\title{
The Impact of a Proactive Chronic Care Management Program on Hospital Admission Rates in a German Health Insurance Society
}

\author{
Brent Hamar, DDS, MPH, Aaron Wells, PhD, William Gandy, EdD, Andreas Haaf, PhD, ${ }^{1}$ \\ Carter Coberley, PhD, James E. Pope, MD, and Elizabeth Y. Rula, PhD ${ }^{1}$
}

\begin{abstract}
Hospital admissions are the source of significant health care expenses, although a large proportion of these admissions can be avoided through proper management of chronic disease. In the present study, we evaluate the impact of a proactive chronic care management program for members of a German insurance society who suffer from chronic disease. Specifically, we tested the impact of nurse-delivered care calls on hospital admission rates. Study participants were insured individuals with coronary artery disease, heart failure, diabetes, or chronic obstructive pulmonary disease who consented to participate in the chronic care management program. Intervention $(n=17,319)$ and Comparison $(n=5668)$ groups were defined based on records of participating (or not participating) in telephonic interactions. Changes in admission rates were calculated from the year prior to (Base) and year after program commencement. Comparative analyses were adjusted for age, sex, region of residence, and disease severity (stratification of 3 [least severe] to 1 [most severe]). Overall, the admission rate in the Intervention group decreased by $6.2 \%$ compared with a $14.9 \%$ increase in the Comparison group $(P<0.001)$. The overall decrease in admissions for the Intervention group was driven by risk stratification levels 2 and 1, for which admissions decreased by $8.2 \%$ and $14.2 \%$ compared to Comparison group increases of $12.1 \%$ and $7.9 \%$, respectively. Additionally, Intervention group admissions decreased as the number of calls increased $(P=0.004)$, indicating a dose-response relationship. These findings indicate that proactive chronic care management care calls can help reduce hospital admissions among German health insurance members with chronic disease. (Population Health Management 2010;13:339-345)
\end{abstract}

\section{Introduction}

C HRONIC DISEASE IS A WORLDWIDE EPIDEMIC that has dramatic consequences for both quality of life and the economy. Patients who do not appropriately manage their chronic condition(s) are prone to acute events that require hospital admissions, a primary driver of health care expenditure. ${ }^{1}$ Further, upon discharge from the hospital, patients often do not receive the necessary education and follow-up to prevent otherwise avoidable readmissions. ${ }^{2-4}$

The widespread need for enhanced care and support measures for individuals with chronic disease led to a new arena of health care-chronic care management (CCM), defined as coordinated health care interventions and communications for populations with conditions that require significant self-care efforts. ${ }^{5}$ Proactive CCM has been widely adopted as a means to improve quality and thereby reduce unnecessary health care utilization and expenditure.

In Germany, legislation enacted in 2002 made disease management (DM) a key component of system-wide health care reform and was a first step toward introducing morbidity as a factor in risk-structure adjusted compensation to sickness funds. ${ }^{6,7}$ In the context of Germany's statutory health insurance (SHI) system, DM has a governmentdefined framework that includes physician adherence to specific evidence-based guidelines and improved coordination among providers. ${ }^{8}$ This approach is somewhat different from the programs provided by private insurers, which typically use multiple modalities (ie, telephone and Internet) to interact with patients as a complementary service to primary care. ${ }^{9}$ Such programs provide additional means to identify patients with unmanaged chronic disease, the flexibility to

\footnotetext{
${ }^{1}$ Center for Health Research, Healthways, Inc, Franklin, Tennessee.

${ }^{2}$ Geschäftsführer, Healthways International $\mathrm{GmbH}$, Hennigsdorf, Germany.
} 
adjust to the needs of the member at any point in time, and reinforcement to support the physician's care plan between visits. $^{6}$

To expand health promotion and care management for its members beyond the scope of DM services available through the SHI system, a German private insurance company initiated a private CCM program in 2008. The program was designed specifically for this insurer using the framework of the domestic programs delivered by a US health management provider and included comprehensive patient-centered services that take into account the full scope of chronic conditions, risk factors, and behaviors to help individuals appropriately treat their conditions and manage their health.

The purpose of this study was to provide an initial evaluation of the therapeutic effect of the proactive chronic care program on hospital admission rates. The study design was constrained by numerous data limitations. First, members of this program were enrolled with the SHI system and private insurance, and only private insurance claims data were available for use in this study; therefore, it was necessary to choose outcome metrics for which we had a complete data set. Additional considerations included the retrospective nature of the study and the absence of data for a comparable nonparticipant group. Within the limits of available data, we chose a within-subject pre-post quasi-experimental design aimed at determining the impact of nurse-delivered care calls-the primary program intervention measure to members of the CCM program-on hospital admission rates.

\section{Methods}

The CCM program was designed to provide holistic support and care to members with chronic disease and was offered in addition to the SHI DM program. The program goals were to improve the quality of medical care and thereby decrease medical expenses and increase member satisfaction. The program focused on educating and empowering members to effectively care for their health with respect to their chronic disease(s). Specifically, the program addressed health-related behaviors, self-care measures, and adherence to standards of care. By helping program members better understand and manage their chronic conditions and providing regular support of their physicians' care plan, the program was designed to prevent or mitigate acute events and related hospitalizations stemming from these conditions.

Administrative medical claims and eligibility data were used to identify eligible members with 1 or more chronic conditions. Specific algorithms used in conjunction with diagnosis codes and drug medication codes were employed to identify eligible members with coronary heart disease (CHD), heart failure, diabetes, or chronic obstructive pulmonary disease (COPD). An active enrollment process was used to recruit members into the program. Members were initially contacted, typically by telephone, and invited to participate in the management program at no cost. Members had to formally agree (opt in) and supply informed consent to participate in the program. Insured members who chose to participate in the program were administered a Quick Screen (QS) survey that collected information about perceived health status, functional status, chronic pain, and number of hospital and emergency room visits in the last year. Mem- bers were categorized into 3 stratification levels using an algorithm that incorporated QS survey responses and available medical documentation of disease conditions. Stratification levels represented estimated disease severity, which ranged from 3 (least severe) to 1 (most severe), and was the primary factor used to determine the type and intensity of interactions with each member. Intervention modalities included telephonic care calls, mailed educational materials, and access to online support. Typically, care calls were scheduled outbound calls by nurses; however, all participants had the option to initiate inbound calls that also were considered in our analysis.

The CCM nurses used proprietary electronic management software to document all member medical information and care call interactions. The nature and content of care call interactions were tailored to the needs of the member, based on current data and historical documentation. The goals of the calls were to support appropriate care choices, provide advice on better self-management practices, and encourage positive health behaviors and adherence to care standards including regular physician visits, disease-appropriate testing, and medication adherence. To achieve these goals, the nurses were trained in the transtheoretical model of behavior change theory and practice, which matches the intervention to an individual's stage in the process of change. ${ }^{10}$ Nurses were given discretion to recategorize a member's risk stratification (and associated frequency of telephonic call support) based on their assessment and clinical judgment of the member's support needs, thus providing the flexibility to adapt the program to unique member needs at the time of interaction. Given this program feature, the risk stratification of any given member was somewhat dynamic in nature, as was the frequency with which he or she received calls.

The program commenced operation on January 2, 2008. The first year of program operation starting from program commencement to January 1, 2009 was defined as the study period (Year 1). The comparison period defined for this study was from January 1, 2007 to January 1, 2008 (Base). Insured members who participated in the CCM program and were documented to have continuous health insurance plan enrollment from the beginning of the Base period to the end of the program Year 1 were eligible for inclusion in the study in alignment with contractually defined program evaluation criteria. Of the 25,064 program participants in Year 1, 24,629 met the continuous enrollment requirement. Of this group, 1642 members who lacked a valid stratification at the beginning of the program were excluded from the analysis, leaving a total of 22,987 members who were included in this study.

The comparison group was chosen in accordance with the limitations of available data. We were unable to identify a suitable comparison group of individuals who were similar to intervened members, but who did not participate in the CCM program and for whom we had the necessary data to conduct the study. In light of this constraint to our design, and as a means to minimize selection bias that might be magnified through comparisons to eligible members who were unwilling to participate in the program, members of both study groups were limited to program members who initially opted to participate in the program and were subject to the same eligibility requirements outlined above. Eligible individuals were grouped into intervened (Intervention; 
$N=17,319)$ and non-intervened (Comparison; $N=5,668$ ) study groups based on their record of participating (or not participating) in program care calls during the study period. Comparison group members received 1 or fewer calls (initial enrollment call only) and Intervention group members received 2 or more calls ( 1 or more care calls following the initial enrollment call).

The retrospective nature of this analysis precluded the use of randomly assigned study groups; consequently, initial analysis showed the two study groups differed on multiple variables in the Base period, most notably the much higher rate of admissions at Base for the Intervention group compared with the Comparison group (Table 1). Based on these observations, a pre-post study design was employed to allow evaluation of the change in admissions as opposed to absolute admission rate during the program year. The primary outcome metrics defined for the study were (1) annualized admission rate per 1000 in Base and Year 1, and (2) percent change in admissions between program periods. Although data tables present results as annualized admission rates, statistical testing was performed at the individual member level. Hospitalizations for which the admission and discharge date were the same were excluded because these likely represent admission for a treatment as opposed to an acute event.

In addition to overall comparisons of admission rate changes between study groups, subgroup analyses were also performed on individuals grouped by stratification level, sex, and disease group to determine the impact of these specific variables on this outcome. Further, smaller subgroups were created that were matched with respect to sex, stratification level, and state of residence to verify that results were broadly consistent across groups that were the same with respect to these variables; statistical testing was not performed on these small subgroups.

Data sources for the study included membership files with demographic, member eligibility, and disease diagnoses, as well as hospital data files that documented hospital admis-

TABle 1. Study Group Comparison

\begin{tabular}{lccc}
\hline & Intervention & Comparison & \\
Descriptive Variable & $\mathrm{N}=17,319$ & $\mathrm{~N}=5668$ & $\mathrm{P}$ value \\
\hline Age, mean & 71.2 & 72.5 & $<0.0001^{1}$ \\
\% Female & 50.8 & 45.6 & $<0.0001^{2}$ \\
\% residing in & 41.6 & 38.1 & $<0.0001^{2}$ \\
$\quad$ Baden-Württemberg & & & \\
$\begin{array}{l}\text { Stratification Group, \% } \\
3\end{array}$ & 29.7 & 41.0 & $<0.0001^{2}$ \\
2 & 46.4 & 37.1 & $<0.0001^{2}$ \\
$\quad$ & 24.0 & 21.9 & $0.0014^{2}$ \\
$\begin{array}{l}\text { Diagnosis 2006, \% } \\
\text { Diabetes }\end{array}$ & 59.3 & 59.7 & $\mathrm{NS}^{2}$ \\
$\quad$ Heart failure & 14.3 & 15.7 & $0.0116^{2}$ \\
$\quad$ Coronary heart disease & 48.0 & 49.5 & $\mathrm{NS}^{2}$ \\
$\quad$ Chronic obstructive & 12.3 & 12.8 & $\mathrm{NS}^{2}$ \\
pulmonary disease & & & \\
$\begin{array}{l}\text { Base admission rate } \\
\text { (per 1000) }\end{array}$ & 505.5 & 255.5 & $<0.0001^{3}$ \\
\hline
\end{tabular}

NS, nonsignificant.

${ }^{1} t$ test; ${ }^{2}$ chi-square test; ${ }^{3}$ Rothman large sample chi-square test. sions of insured members from 2006 through the end of 2009. Records from the CCM program included documented dates of all successful calls during which the member interacted with a CCM nurse. Members of both study groups were categorized based on their initial stratification level assignment in program stratification files at the time of enrollment. All data were de-identified to protect the privacy of participants.

Univariate statistical comparisons of aggregate-level study group attributes were performed using the Student $t$ test for continuous variables and the chi-square test for categorical variables. Multivariate analyses using a generalized linear model were conducted to determine whether the intervention was a statistically significant predictor of a change in admissions from Base to Year 1 while controlling for differences in age, sex, state of residence, and stratification level. Additionally, a 3-level ordinal variable indicating the member's admission change status from Base to Year 1 (reduction, no change, increase) was used in ordinal logistic regression analyses to determine the impact of the intervention on study members' admission change status while controlling for covariates. This approach was also used to test the doseresponse relationship between number of calls and hospital admission rate changes. Data manipulation and analysis was performed using SAS 9.2 statistical software (SAS Institute Inc., Cary, NC).

\section{Results}

A comparison of all admissions (primary and readmissions) between Base and Year 1 demonstrated that there was an increase in the rate of admission among Comparison group members and a decrease in the rate among members of the Intervention group (Table 2), and that the difference between the groups was significant when controlling for other covariates $(P=0.0002)$.

An evaluation of members grouped by initial stratification level found that the mean number of calls to Intervention group members during Year 1 was as follows: level 3, 3.32 calls; level 2, 6.15 calls; and level 1, 10.11 calls. Admission rates, when compared within stratification levels, demonstrated that the overall decrease in Intervention group admission rate was driven by stratification levels 2 and 1 , which were the groups with the highest levels of program participation. Intervention and Comparison group members were also divided into subgroups based on their stratification level, sex, and region of residence to allow for comparisons of subsets of members who were the same with respect to these characteristics. The results of this analysis showed that the relationship between the Intervention and Comparison group exhibited in the overall comparison was consistent in nearly all matched group comparisons (data not shown), indicating that the result was consistent with respect to these variables.

Both female and male members in the Intervention group showed significant decreases in admission rate compared to Comparison group members of the same sex, although females had a greater reduction (Table 3). Intervention group females achieved greater overall reductions in admissions than males with respect to Comparison group members of the same sex. However, after controlling for other covariates, results from multivariate statistical modeling indicated that 
Table 2. Admission Rate Changes from Base to Year 1

\begin{tabular}{|c|c|c|c|c|c|c|}
\hline $\begin{array}{l}\text { Stratification } \\
\text { Level }\end{array}$ & Study Group & $\mathrm{N}$ & $\begin{array}{l}\text { Base Admission } \\
\text { Rate (per 1000) }\end{array}$ & $\begin{array}{l}\text { Year } 1 \text { Admission } \\
\text { Rate (per 1000) }\end{array}$ & $\begin{array}{l}\text { Percent } \\
\text { Change }\end{array}$ & P Value \\
\hline- & Comparison & 5668 & 255.5 & 293.6 & $14.9 \%$ & 0.0002 \\
\hline- & Intervention & 17,319 & 505.5 & 474.3 & $-6.2 \%$ & \\
\hline \multirow[t]{2}{*}{3} & Comparison & 2326 & 163.8 & 210.7 & $28.6 \%$ & \multirow[t]{2}{*}{ NS } \\
\hline & Intervention & 5140 & 229.2 & 289.9 & $26.5 \%$ & \\
\hline \multirow[t]{2}{*}{2} & Comparison & 2101 & 259.4 & 290.8 & $12.1 \%$ & \multirow[t]{2}{*}{0.0121} \\
\hline & Intervention & 8029 & 459.1 & 421.6 & $-8.2 \%$ & \\
\hline \multirow[t]{2}{*}{1} & Comparison & 1241 & 420.6 & 453.7 & $7.9 \%$ & \multirow[t]{2}{*}{$<0.0001$} \\
\hline & Intervention & 4150 & 937.6 & 804.8 & $-14.2 \%$ & \\
\hline
\end{tabular}

NS, nonsignificant.

${ }^{1}$ Rates are presented for qualitative comparisons only and were not used for statistical testing.

${ }^{2}$ Statistical testing employed multivariate ordinal logistic regression analysis of individual member level data.

sex was not a significant predictor of admission rate change from Base to Year $1(P=0.2019)$.

In a comparison of admission rate changes by disease group, shown in Table 4, the heart failure Intervention population experienced the greatest absolute and relative reduction in admission rates. Intervention group members with diabetes and COPD trended toward a reduction in admissions, but the difference between the groups did not achieve statistical significance.

As a final analysis, we investigated a dose-response relationship between number of care calls and change in admissions. Figure 1 presents the percent change in the admission rate from Base to Year 1 for groups defined by the number of successful calls in Year 1. Overall intervention group results show a progressively greater decrease in admission rate from Base to Year 1 between members who received 2 calls through members who received 6-7 calls. Results from multivariate modeling indicated that number of calls, as categorized in Figure 1, is a significant predictor of a change in admission rate from Base to Year 1, while controlling for other covariates $(P<0.0001)$.

\section{Sensitivity testing}

Because of the differences in admission rate between the two study groups at baseline, sensitivity analyses were performed to ensure that the results were not an artifact of outliers or regression to the mean. First, a core subpopulation of members with an admission count change ranging from -2 to $+2(N=22,074$, or $96.0 \%$ of the total study population) were evaluated to remove effects of those members with a dramatic increase or decrease in admissions (ie, potential outliers). In this subset analysis, the admission rate decreased by $6.9 \%$ in the Intervention group and increased by $14.2 \%$ in the Comparison group $(P<0.001)$. To further ensure that the results reflected actual program impact, the analysis was next limited to a subpopulation of members with either no change in the number of admissions across the study periods, or an increase in admissions. This 1-sided evaluation revealed that the increase in admission rate was 13\% lower in the Intervention group than in the Comparison group (data not shown).

\section{Discussion}

CCM, often referred to as DM, as typically defined in the United States, is a patient-centered approach to supporting self-efficacy, healthy behavior, and appropriate treatment for the management of the full range of chronic conditions and comorbidities of each member. This approach can be differentiated from the DM programs of the German SHI system that implement standardized care guidelines and protocols into primary care for the purpose of improving the quality of care, and also to provide a means of risk-structure adjusted compensation to insurers. These SHI DM programs are tightly regulated and controlled, providing only limited scope for competitive approaches and are limited by requiring physician participation. ${ }^{11}$

In this study, we evaluated the impact of a CCM program, developed and administered by a US health management provider, on the hospitalization rates of members of a private insurer in Germany with chronic disease(s). The primary intervention of the program was telephonic care calls delivered by trained nurse-counselors. All study participants

Table 3. Change in Admissions per 1000 from Base to Year 1 by Sex

\begin{tabular}{|c|c|c|c|c|c|c|}
\hline \multirow[b]{2}{*}{ Sex } & \multirow[b]{2}{*}{ Study Group } & \multirow[b]{2}{*}{$\mathrm{N}$} & \multicolumn{3}{|c|}{ Admission Rate (Per 1000) ${ }^{1}$} & \multirow[b]{2}{*}{ P Value } \\
\hline & & & Base & Year 1 & $\% \Delta$ & \\
\hline \multirow[t]{2}{*}{ Female } & Comparison & 2584 & 244.6 & 294.1 & $20.3 \%$ & \multirow[t]{2}{*}{0.0077} \\
\hline & Intervention & 8791 & 473.6 & 439.4 & $-7.2 \%$ & \\
\hline \multirow[t]{2}{*}{ Male } & Comparison & 3084 & 264.6 & 293.1 & $10.8 \%$ & \multirow[t]{2}{*}{0.0094} \\
\hline & Intervention & 8528 & 538.5 & 510.3 & $-5.2 \%$ & \\
\hline
\end{tabular}

${ }^{1}$ Rates are presented for qualitative comparisons only and were not used for statistical testing.

${ }^{2}$ Statistical testing employed multivariate ordinal logistic regression analysis of individual member level data. 
Table 4. Change in Admissions per 1000 from Base to Year 1 by Disease Group

\begin{tabular}{|c|c|c|c|c|c|c|}
\hline \multirow[b]{2}{*}{ Diagnosis } & \multirow[b]{2}{*}{ Study Group } & \multirow[b]{2}{*}{$\mathrm{N}$} & \multicolumn{3}{|c|}{ Admission Rate (per 1000) } & \multirow[b]{2}{*}{ P Value $^{2}$} \\
\hline & & & Base & Year 1 & $\% \Delta$ & \\
\hline Diabetes & $\begin{array}{l}\text { Comparison } \\
\text { Intervention }\end{array}$ & $\begin{array}{r}3381 \\
10,265\end{array}$ & $\begin{array}{l}215.0 \\
451.0\end{array}$ & $\begin{array}{l}262.6 \\
456.2\end{array}$ & $\begin{array}{r}22.1 \% \\
1.1 \%\end{array}$ & NS \\
\hline Heart failure & $\begin{array}{l}\text { Comparison } \\
\text { Intervention }\end{array}$ & $\begin{array}{r}888 \\
2477\end{array}$ & $\begin{array}{l}330.0 \\
706.9\end{array}$ & $\begin{array}{l}418.9 \\
635.0\end{array}$ & $\begin{array}{r}27.0 \% \\
-10.2 \%\end{array}$ & 0.0062 \\
\hline CHD & $\begin{array}{l}\text { Comparison } \\
\text { Intervention }\end{array}$ & $\begin{array}{l}2804 \\
8311\end{array}$ & $\begin{array}{l}313.5 \\
584.5\end{array}$ & $\begin{array}{l}370.5 \\
560.6\end{array}$ & $\begin{array}{r}18.2 \% \\
-4.1 \%\end{array}$ & 0.0072 \\
\hline COPD & $\begin{array}{l}\text { Comparison } \\
\text { Intervention }\end{array}$ & $\begin{array}{r}724 \\
2131\end{array}$ & $\begin{array}{l}331.5 \\
629.3\end{array}$ & $\begin{array}{l}346.7 \\
595.0\end{array}$ & $\begin{array}{r}4.6 \% \\
-5.4 \%\end{array}$ & NS \\
\hline
\end{tabular}

NS, nonsignificant; CHD, coronary heart disease; COPD, chronic obstructive pulmonary disease.

${ }^{1}$ Rates are presented for qualitative comparisons only and were not used for statistical testing.

${ }^{2}$ Statistical testing employed multivariate ordinal logistic regression analysis of individual member level data.

had access to both SHI DM and the program evaluated here; therefore, the goal of the study was to determine the incremental value of telephonic care calls as a part of this program. The results of our analysis demonstrated that members of the program who engaged in these care calls had a significant reduction in hospital admissions during the first year of the program as compared to members who enrolled in the program but did not interact beyond the initial enrollment call. This finding indicates that participation in care calls reduces the likelihood of inpatient admission in a population that is diverse with respect to disease diagnosis and severity. To our knowledge, this is the first large-scale study of a telephonic CCM program in Germany and provides initial evidence that such scalable programs can be beneficial when delivered in the context of the German health care system.
A challenge we encountered in this retrospective evaluation was the identification of a nonparticipant comparison group that was equivalent to the Intervention group, an issue that is common in the evaluation of CCM programs. ${ }^{12} \mathrm{Be}-$ cause of the absence of available data from a similar group of individuals who met program eligibility criteria but who were not offered the program, we were limited to constructing the comparison group from members of the health insurance plan who either opted out of the program or who enrolled in the program but did not actively participate. We anticipated that by limiting the study to insured members who actively opted to enroll in the program, the impact of selection bias would be mitigated; however, the differences in the study groups' characteristics-the most noteworthy of which was Base year admission rate-seem to indicate that members self-selected how much (or little) they wanted to

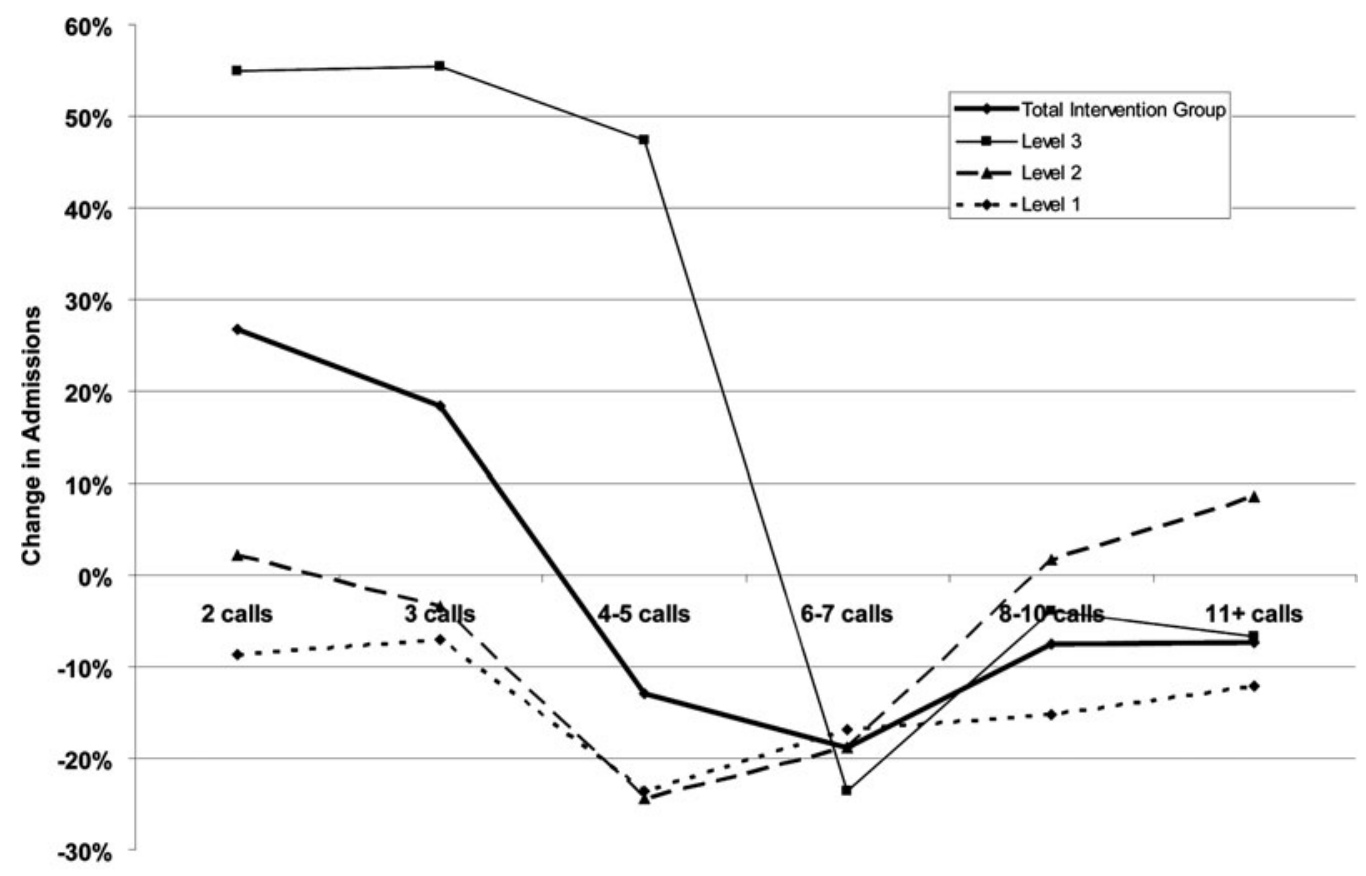

Intervention Group Members by Number of Calls

FIG. 1. Percent change in Intervention group admission rates by number of care calls during Year 1. 
interact with the CCM program nurse staff. However, the fact that calls were attempted more frequently for members with greater disease severity also may be a likely cause of the difference between the groups, as evidenced by the fact that members with greater disease severity and recent health care utilization were more likely to have taken a care call. Although these apparent sources of bias introduce a challenge to evaluating program outcomes, the greater severity level of the Intervention group demonstrates that the program effectively reaches and engages the members of the population who are in greatest need of support.

Because of the difference in morbidity between the two study groups and an insufficient pool of individuals in the Comparison group to allow for exact or propensity score matching of acceptable fidelity ${ }^{13}$ we controlled for the differences between the two groups statistically using regression modeling that adjusts for observable covariates. To improve comparability, we evaluated admission rate changes in subgroups matched on stratification level, sex, and state of residence and found that results were consistent for nearly all subgroups, which provided additional confidence in our result. Further, we compared the groups with respect to stratification level, sex, and disease as separate factors to understand the relationship of these variables to the outcome of interest.

In our evaluation of outcomes by stratification level, results demonstrated that there were significant decreases in admission rates for the Intervention group in stratification levels 2 and 1 compared with the Comparison group; the study groups had similar results among stratification level 3 members. These lower severity members in both study groups had noteworthy increases in admission rates, indicating the presence of latent risk among members of this group that was not identified in the method of risk stratification. The program has since introduced a predictive model that identifies risk stratification level 3 members with a high likelihood of hospitalizations such that their call frequency can be increased and improved outcomes can be achieved. Future analysis will determine the effectiveness of this enhanced targeting approach.

Analysis of specific disease populations indicated that members diagnosed with heart failure achieved greater reductions in admissions (when compared to Comparison group results) than other disease groups. Previous research has established a direct link between nonadherence to diet and medications with an increased likelihood of hospitalization and mortality for patients with heart failure. ${ }^{14}$ Our results suggest that heart failure patients who are educated about the warning signs of deteriorating health and who are encouraged to adhere to established standards of care are more likely to take the necessary steps to prevent acute events, such as fluid overload, ${ }^{15}$ that result in hospitalization. This was a particularly favorable result in light of the fact that heart failure is the predominant reason for hospitalization in Germany ${ }^{16}$ and the rate of heart failure admissions is higher than average compared with other industrialized countries. $^{17}$

As a whole, the differential impact of the program on members of different disease groups could be explained in many ways, but there are 2 primary hypotheses for this result. First, heart failure and CHD may be more sensitive to shortterm changes in admissions as a result of the intervention than diabetes or COPD, and longer term studies would reveal significant improvements in these two disease groups. Second, the evaluated program may be more efficacious for certain diseases than for others, or for impacting specific outcomes over others. COPD had the lowest magnitude of impact of any of the disease groups, consistent with the fact that there is mixed evidence concerning the effectiveness of COPD interventions to improve hospitalizations. ${ }^{18,19}$ Future studies should evaluate interventions of longer duration across these diseases, and evaluate the specific treatment elements that are most efficacious for a given disease.

Our evaluation of a potential dose-response relationship between care calls and reductions in admissions found that participation in an increasing number of calls (up to some point) results in a more positive impact on percent change in admissions, after which the effect is saturated. This phenomenon illustrates that instruction on basic care, adherence to medications, and physician follow-up, as advocated during the care calls, may help a patient improve up to a certain point, after which additional contact may not further reduce the event rate but instead help to maintain the improvement. It should be noted that these results are confounded by the fact that members who developed or maintained high disease severity during the course of the program were targeted for more calls. Therefore, the admission rates of members with more calls than the average for their group are expected to reflect a greater likelihood of an acute event, which would warrant the additional calls. Despite these factors that complicate the interpretation of the data, our statistical analysis that accounted for covariates demonstrated that, as a whole, there was a significant relationship between number of calls and decline in admission rate. This result is consistent with previous research demonstrating that an increased frequency of care calls is associated with greater adherence to standards of care among DM participants. ${ }^{20,21}$

The primary limitation of this study was the use of a Comparison group that had a significantly lower rate of admissions during the Base year compared with the Intervention group, which introduces the potential for regression to the mean. This statistical phenomenon describes the propensity of extreme observations to naturally return to a predictable mean value. $^{22}$ Further, the admission rate variables had a wide distribution, suggesting that outliers may have impacted the results. We performed sensitivity analyses to help gauge and account for the potential impact of both of these factors on analysis results. Because a significant positive impact of the intervention was demonstrated using only the subset of the study population with a change in admission count of 2 or less and in a 1-sided analysis of the subset with either no change or an increase in admissions, we conclude that the results of the analysis were not primarily driven by either regression to the mean or population outliers.

Results from this study raise other research questions that warrant future investigation. First, although we were able to demonstrate that care calls have an impact on admissions, we have not yet explored the impact of calls on other outcomes of interest, particularly the mediators of admission reduction, health care costs, quality of life, and member satisfaction. Although we evaluated outcomes with respect to severity level, disease, and sex, more work should be done to explore specific member characteristics that might improve the specificity with which the intensity of intervention 
can be matched to each member's status. This study also revealed that there are numerous individuals who opted into the program but then did not interact with nurses. Because this group has poor outcomes compared with members who do participate in care calls, it is likely that overall program effectiveness would improve if more individuals were actively engaged in the program. Future work should be done to investigate means to improve engagement and the impact of higher engagement on program outcomes.

\section{Limitations}

The retrospective nature of this study and limited data availability precluded the creation of a comparison group that was more equivalent to the Intervention group and the development of alternative proxies for morbidity level. Further, the independent and dependent variables in scope for this study were limited by the data that were available for analysis.

\section{Conclusions}

In this initial evaluation of a US proactive CCM program delivered in the context of the German health care system, our findings demonstrate that care calls to privately insured individuals with chronic disease succeeded in reducing hospital admission rates compared to members who did not engage in these calls. The fact that the population was not altogether naïve to DM interventions, as delivered through $\mathrm{SHI}$, makes the short-term impact of this program particularly noteworthy. Although beyond the scope for analysis in this study, we anticipate that the identified reduction in hospitalizations was associated with a decrease in medical expenditures and an increase in health-related quality of life among Intervention group members. The results of this study demonstrate the effectiveness of proactive CCM programs at helping individuals better manage their chronic conditions to avoid inpatient hospital admissions.

\section{Author Disclosure Statement}

Drs. Hamar, Wells, Gandy, Haaf, Coberley, Pope, and Rula are employed by Healthways and own stock in the company.

The study was funded by Healthways, the provider of the chronic care management program reported on in this study.

\section{References}

1. Burchardi H, Schuster HP, Zielmann S. Cost containment: Europe. Germany. New Horiz 1994;2:364-374.

2. Benbassat J, Taragin M. Hospital readmissions as a measure of quality of health care: Advantages and limitations. Arch Intern Med 2000;160:1074-1081.

3. Makaryus AN, Friedman EA. Patients' understanding of their treatment plans and diagnosis at discharge. Mayo Clin Proc 2005;80:991-994.

4. Maniaci MJ, Heckman MG, Dawson NL. Functional health literacy and understanding of medications at discharge. Mayo Clin Proc 2008;83:554-558.

5. DMAA: The Care Continuum Alliance. DMAA definition of disease management. Available at: http://www.carecontinuum .org/dm_definition.asp. Accessed October 28, 2010.
6. Pittman PM, Arnold SB, Schlette S. Care management in Germany and the US: An expanded laboratory. Health Care Financ Rev 2005;27:9-18.

7. Greiner W. [Health economic evaluation of disease management programs]. Bundesgesundheitsblatt Gesundheitsforschung Gesundheitsschutz 2006;49:34-39.

8. Busse R. Disease management programs in Germany's statutory health insurance system. Health Aff (Millwood) 2004;23:56-67.

9. Schrijvers G. Disease management: A proposal for a new definition. Int J Integr Care 2009;9:e06.

10. Prochaska JO, Velicer WF. The transtheoretical model of health behavior change. Am J Health Promot 1997;12:38-48.

11. Kottmair S, Frye C, Ziegenhagen DJ. Germany's disease management program: Improving outcomes in congestive heart failure. Health Care Financ Rev 2005;27:79-87.

12. Alliance DTCC. Outcomes Guidelines Report. Volume 4. Washington, DC: DMAA; 2009.

13. Shadish WR, Cook TD, Campbell DT. Experimental and Quasi-Experimental Designs for Generalized Causal Inference. New York: Houghton Mifflin Company; 2002.

14. Fonarow GC, Abraham WT, Albert NM, et al. Factors identified as precipitating hospital admissions for heart failure and clinical outcomes: Findings from OPTIMIZE-HF. Arch Intern Med 2008;168:847-854.

15. Yu CM, Wang L, Chau E, et al. Intrathoracic impedance monitoring in patients with heart failure: Correlation with fluid status and feasibility of early warning preceding hospitalization. Circulation 2005;112:841-848.

16. Neumann T, Biermann J, Erbel R, et al. Heart failure: The commonest reason for hospital admission in Germany: Medical and economic perspectives. Dtsch Arztebl Int. 2009;106:269-275.

17. Organisation for Economic Co-operation and Development. OECD health at a glance 2009: Key findings for Germany. Available at: http://www.oecd.org/document/2/0,3343, en_33873108_33873402_44220930_1_1_1_1,00.html. Accessed October 27, 2010.

18. Peytremann-Bridevaux I, Staeger P, Bridevaux PO, Ghali WA, Burnand B. Effectiveness of chronic obstructive pulmonary disease-management programs: Systematic review and meta-analysis. Am J Med 2008;121:433-443.e4.

19. Clark NM, Dodge JA, Partridge MR, Martinez FJ. Focusing on outcomes: Making the most of COPD interventions. Int J Chron Obstruct Pulmon Dis 2009;4:61-77.

20. Coberley CR, McGinnis M, Orr PM, et al. Association between frequency of telephonic contact and clinical testing for a large, geographically diverse diabetes disease management population. Dis Manag 2007;10:101-109.

21. Coberley C, Hamar B, Gandy B, et al. Impact of telephonic interventions on glycosylated hemoglobin and low-density lipoprotein cholesterol testing. Am J Manag Care 2007;13: 188-192.

22. Morton V, Torgerson DJ. Effect of regression to the mean on decision making in health care. BMJ 2003;326:1083-1084.

Address correspondence to: Elizabeth Y. Rula, PhD

Center for Health Research, Healthways, Inc 701 Cool Springs Boulevard Franklin, TN 37067

E-mail: elizabeth.rula@healthways.com 
